\title{
Social mice seeking circuits
}

\author{
Hu et al. show that the posterodorsal medial amygdala selectively controls social-reward seeking through its \\ intersection with canonical dopaminergic reward circuits. To identify this circuitry, the authors developed an \\ elegant new affiliative social operant procedure that separates social interaction from social-reward seeking.
}

\author{
Eric R. Szelenyi, Nastacia L. Goodwin and Sam A. Golden
}

T he COVID-19 pandemic has drastically altered the world's daily routines, highlighting the importance of sociality for mental health. Now more than ever, outside of our immediate pods, every social interaction is weighted against potential harm. Yet many understandably find themselves driven, against better judgment and medical consensus, to seek the presence and comfort of others. In this issue of Nature Neuroscience, Hu et al. ${ }^{1}$ identify a neural pathway that mediates affiliative social-reward seeking in the rodent brain. They employ neural circuit recording and manipulation methods in conjunction with an elegantly designed social operant procedure to identify an upstream social circuit node that modulates the canonical reward circuitry. Specifically, they find that GABAergic neurons in the posterodorsal part of the medial amygdala (MeApd) form a crucial 'social node' that drives dopamine (DA) release in the nucleus accumbens (NAc) via the medial preoptic nucleus (MPN) (Fig. 1a).

The field of behavioral neuroscience is rapidly evolving, driven primarily by genetic and technological advances, but the parallel development of advanced behavioral procedures has lagged. This has been countered by a call to refocus technical advancements within the bounds of interpretable behavior, where tasks are not merely reductionist tools for circuit perturbation but rather a gestalt a priori framework that includes diligent design and the collection of thorough behavioral metrics, to provide holistic mechanistic insights ${ }^{2}$. This lag has been especially apparent with the common use of classical social behavior assays, such as the three-chamber sociality and conditioned place preference tests, which use primary metrics like passive exploratory locomotion rather than discrete contingent action.

To circumvent this issue, the authors developed a fully automated, closed-loop operant procedure in which adult male or female mice learned to nose poke one of two ports (the 'social port') to obtain access to a novel same-sex juvenile conspecific through a barrier screen (Fig. 1b). In addition, a
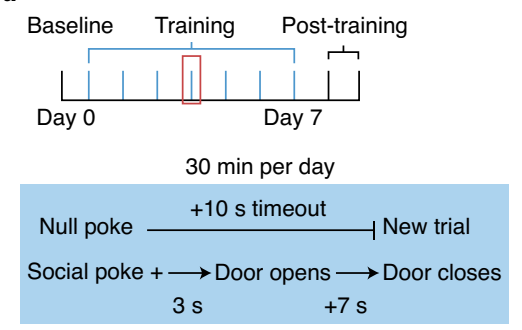

b
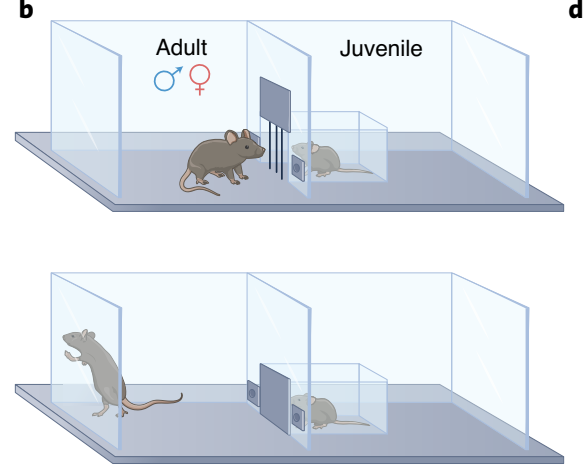

d
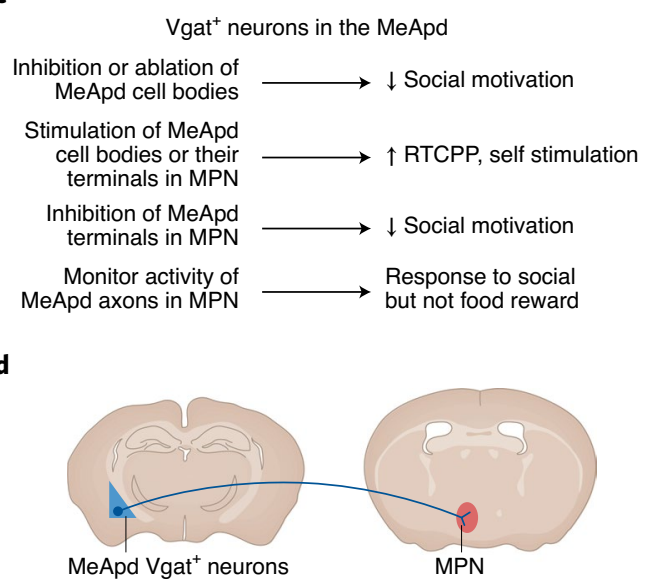

Fig. 1| Graphical summary of Hu et al. a, Timeline of behavioral training and novel operant nose poke task. $\mathbf{b}$, When a mouse nose-pokes the social port, this triggers opening of the guillotine door to allow social interaction with a juvenile through a grate. $\mathbf{c}$, Summary of key findings demonstrating the importance of Vgat $^{+}$neurons in social seeking. d, Stimulation of ggat $^{+}$neurons in the MeApd projecting to the MPN (top) increases operant responding in the task, whereas inhibition of this pathway results in lower operant responding (bottom). RTCPP, real-time conditioned place preference.

unlike the three-chambered sociality and conditioned place-preference tests in which social interactions are forced, this procedure enabled the authors to study social-reward seeking under purely volitional conditions triggered by active operant actions. This type of approach had previously been used to explore the neurocircuitry of other forms of social motivation, such as aggression-reward seeking ${ }^{3}$, and are an important advance in the study of affiliative social motivation.

The MeApd is a critical node in aggression $^{4}$ and parenting ${ }^{5}$ circuits, and the authors wondered whether it also plays a role in regulating affiliative social-reward behavior. Focusing on GABAergic cells in the MeApd, the authors used a vGAT-Cre (a GABAergic cell type) driver line enabling local cell-type-specific manipulations via adeno-associated virus (AAV) transgene expression. They found that ablation or acute optogenetic inhibition of these cells attenuated operant nose-poking for the social port in the operant social reward seeking test. This result demonstrated a necessary role of MeApd GABAergic cells in the reinforcement and maintenance of social reward.

Conversely, the authors demonstrated that optogenetic activation of MeApd GABAergic neurons was sufficient to induce social-reward seeking: mice developed real-time conditioned place preference for a chamber paired with optogenetic stimulation of MeApd GABAergic neurons, and they freely chose optogenetic self-stimulation when given the option between a stimulation-paired port and an unpaired port. Additionally, 
in the operant procedure, when one port was coupled to optogenetic stimulation of MeApd GABAergic neurons (rather than presentation of another mouse), the mice developed a strong preference for that port over the control port.

How does activation of GABAergic neurons in the MeApd modulate canonical reward processing? The MeApd does not directly innervate the NAc. However, it does project to the MPN, which in turn innervates the classic mesolimbic dopaminergic ventral tegmental area (VTA)-to-NAc pathway. Previous studies have shown a role for MPN-VTA connectivity ${ }^{6-8}$ and activity in social reward ${ }^{6}$. Additionally, the MPN is involved in social behaviors including parenting $^{7-9}$, mating ${ }^{10}$, and aggression ${ }^{11}$. The authors therefore examined whether the MeApd regulates affiliative social reward behavior through an MPN-VTA-NAc pathway.

First, they assessed whether social behavior triggers DA release in the NAc, a canonical reward hub. Specifically, they expressed dLight-a genetically encoded DA sensorin the NAc and quantified fluorescence transients using fiber photometry. DA dynamics correlated with several components of the operant task, such as onset and duration of access (through a barrier) to a social partner, and with individual social-port nose pokes. Moreover, optogenetic stimulation of MeApd GABAergic neurons induced a strong time-locked increase in dLight fluorescence, indicating that activation of these neurons in the MeApd is sufficient to drive DA release in the NAc. In addition, the authors found increases in dLight fluorescence in the NAc when mice were freely interacting with other mice. Taken together, these results indicate that social interaction and operant social reward seeking induce the release of DA in the NAc.

Next, the authors assessed whether this effect on DA release was mediated by MPN neurons. Mice showed both real-time place preference and operant intracranial self-stimulation for optogenetic activation of MeApd GABAergic terminals in the MPN. In the operant task, mice developed a preference for the nose port associated with terminal stimulation and increased the number of operant responses across trials, suggesting that terminal stimulation is sufficient to drive a learned operant response. Importantly, similar stimulation of MeApd terminals located in the ventral premammilary nucleus-an adjacent projection targetdid not reproduce these effects, suggesting behavioral specificity of the MeApd-MPN pathway. Combined, these data position MeApd GABAergic neurons as the driver of the MPN-VTA pathway previously implicated in social reward ${ }^{6}$ (Fig. 1d).

The authors then showed that MPN-projecting MeApd neurons are required for driving social reward. Specifically, they delivered a retrograde Cre-recombinase into the MPN that allowed for expression of a Cre-dependent inhibitory optogenetic construct in the MeApd. Inhibiting this pathway reduced nose pokes for the social port in the social operant procedure and substantially reduced the amount of time the mice spent in a social-paired versus non-social-paired chamber during a three-chamber place preference assay. Importantly, the MPN-projecting neuronal population in the MeApd was $~ 85 \%$ GABAergic, suggesting that manipulations in the vGAT-Cre mice mentioned above were predominantly affecting MPN projection neurons. A key control experiment showed that the MeApd-MPN pathway does not mediate food reward or food-reward seeking behavior.

Finally, the authors strengthened the evidence for the MeApd as a reward node by examining whether manipulations of MeApd neuron activity could drive mice to overcome aversion to anxiogenic environments. Using standard tests of anxiety-like behavior (open field and elevated plus maze), the authors found that stimulation of GABAergic MPN-projecting MeApd terminals increased time spent in anxiogenic regions of the testing apparatus. These experiments suggest that MeApd-derived reward is strong enough to overcome a negative valence or reduce anxiogenic influences.

In summary, $\mathrm{Hu}$ et al. demonstrate the benefits of pairing novel operant social behavioral procedures with advanced neural recording and manipulation methods for identifying the circuits regulating affiliative operant social behavior. They show that GABAergic MeApd neurons upstream of the MPN-VTA-NAc pathway drive social reward. Notably, this finding begins to explain how the apparently divergent functions of the MeApd in social behavior (e.g., aggression in males, sexual responses in females) may reflect its more general role as a modulator of social reward seeking across a spectrum of behaviors.

It is important to note that while operant approaches enable the separation of social reward consumption and seeking, they remove naturalistic investigation and ethologically relevant social interactions. These influences are critical for understanding the context within which social choices are made. Recent advances in computational neuroethology ${ }^{12}$, open-source machine-learning-based tracking ${ }^{13,14}$, and analytical tools optimized for complex social behavior ${ }^{15}$ make such in-depth investigations more accessible to an array of behavioral neuroscientists. The future convergence of social operant procedures with computational neuroethological analysis of freely interacting animals holds tremendous promise for enhancing our ability to understand the neural basis of complex social interactions and why individuals choose to seek or avoid certain interactions. Hu et al'.s ${ }^{1}$ identification of the MeApd as a critical upstream pathway-modulating canonical reward circuitry within the context of affiliative social behavior provides an exciting new jumping-off point for these studies.

Eric R. Szelenyi $i^{1,2}$, Nastacia L. Goodwin ${ }^{1,3}$ and Sam A. Golden (iD) 1,2,3凶

${ }^{1}$ University of Washington, Department of Biological Structure, Seattle, WA, USA. ${ }^{2}$ University of Washington, Center of Excellence in Neurobiology of Addiction, Pain, and Emotion (NAPE), Seattle, WA, USA. ${ }^{3}$ University of Washington, Graduate Program in Neuroscience, Seattle, WA, USA.

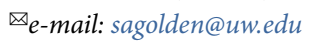

Published online: 7 May 2021

https://doi.org/10.1038/s41593-021-00861-1

References

1. Hu, R. K. et al. An amygdala-to-hypothalamic circuit for social reward. Nat. Neurosci. https://doi.org/10.1038/s41593-021-00828-2 (2021).

2. Krakauer, J. W., Ghazanfar, A. A., Gomez-Marin, A., MacIver, M. A. \& Poeppel, D. Neuroscience needs behavior: correcting a reductionist bias. Neuron $93,480-490$ (2017)

3. Golden, S. A., Jin, M. \& Shaham, Y. Animal models of (or for) aggression reward, addiction, and relapse: behavior and circuits. J. Neurosci. 39, 3996-4008 (2019).

4. Unger, E. K. et al. Medial amygdalar aromatase neurons regulate aggression in both sexes. Cell Rep. 10, 453-462 (2015).

5. Chen, P. B. et al. Sexually dimorphic control of parenting behavior by the medial amygdala. Cell 176, 1206-1221.e18 (2019).

6. McHenry, J. A. et al. Hormonal gain control of a medial preoptic area social reward circuit. Nat. Neurosci. 20, 449-458 (2017).

7. Kohl, J. et al. Functional circuit architecture underlying parental behaviour. Nature 556, 326-331 (2018).

8. Fang, Y.-Y., Yamaguchi, T., Song, S. C., Tritsch, N. X. \& Lin, D. A Hypothalamic midbrain pathway essential for driving maternal behaviors. Neuron 98, 192-207.e10 (2018).

9. Zhang, G.-W. et al. Medial preoptic area antagonistically mediates stress-induced anxiety and parental behavior. Nat. Neurosci. 24, 516-528 (2021)

10. Wei, Y.-C. et al. Medial preoptic area in mice is capable of mediating sexually dimorphic behaviors regardless of gender. Nat. Commun. 9, 279 (2018)

11. Lin, D. et al. Functional identification of an aggression locus in the mouse hypothalamus. Nature 470, 221-226 (2011).

12. Datta, S. R., Anderson, D. J., Branson, K., Perona, P. \& Leifer, A. Computational neuroethology: a call to action. Neuron 104, 11-24 (2019)

13. Mathis, A. et al. DeepLabCut: markerless pose estimation of user-defined body parts with deep learning. Nat. Neurosci. 21, $1281-1289$ (2018)

14. Pereira, T. D. et al. SLEAP: multi-animal pose tracking. Preprint at bioRxiv https://doi.org/10.1101/2020.08.31.276246 (2020).

15. Nilsson, S. R. et al. Simple Behavioral Analysis (SimBA) - an open source toolkit for computer classification of complex social behaviors in experimental animals. Preprint at bioRxiv https://doi. org/10.1101/2020.04.19.049452 (2020).

Competing interests

The authors declare no competing interests. 